\title{
Properties of the volume phase in the layerwise growth
}

\author{
R.Brodskii, A.V.Tur, V.V.Yanovsky \\ State Scientific Institution "Institute for Single Crystals" National \\ Academy of Sciences of Ukraine, Lenina av. 60, 61001 Kharkiv, Ukraine \\ V. Karazin Kharkiv National University, \\ Svobody av., 4, 61000, Kharkiv, Ukraine \\ Universite de Toulouse [UPS], CNRS, Institut de Recherche en \\ Astrophysique et Planetologie, 9 avenue du Colonel Roche, BP 44346, \\ 31028 Toulouse Cedex 4, France
}

\section{Received September 18, 2014}

\begin{abstract}
Assuming statistical properties of phases in the layer are specified, some properties of the volume phase permeating many layers studied. In this paper, we consider analytically the case of one island in the layer and numerically the case of larger and a random number of islands of other phase. The distribution function of the phases overlapping area is obtained. Universal behavior of the distribution function in the small-scale field is established.
\end{abstract}

Предполагая заданными статистические свойства фаз в слое, изучены некоторые свойства объемной фазы пронизывающей много слоев. Аналитически рассмотрен случай одного острова в слое и численно при большем и случайном числе островков другой фазы. Получена функция распределения площади перекрытия фаз. Установлен универсальный характер поведения этой функции распределения в мелкомасштабной области.

Властивості об'ємної фази при пошаровому рості. Р.є.Бродсъкий, А.В.Тур, В.В.Яновський.

Припускаючи заданими статистичні властивості фаз у шарі, вивчено властивості об'ємної фази, яка пронизує багато шарів. Аналітично розглянуто випадок одного острову нової фази у шарі й чисельно у випадку більшої їх кількості. Отримано функцію розподілу площі перекриття фаз. Встановлено універсальний характер залежності цієї функції розподілу в дрібномасштабній області.

\section{Introduction}

In the process of layerwise material growth often forms a layered structure usually complex phase composition. Such mechanisms of crystals and film growth are well known [1] - [4]. In this paper we consider the mechanism of forming the volume phase by way of overlap of a certain phase islands lying in different layers. Overlapping of these islands forms the volume phase of uniform composition crossing a number of intersecting layers. Follow it is convenient to talk about «transparent» phase and the formation of transparency windows, this can be understood as any property specifies the considered phase. Thus suggesting statistical properties of some islands in the layer phase are known, it is required to determine the measure of the volume phase or determine criteria for its absence. As physically convenient features we select in the work probability distribution of phase centers in layer, and its size distribution. The form of phase in the layer is assumed, for simplicity, circular. It also assumes independence of probabilistic characteristics of phases in each layer. As observed magnitude considered area of the region of transparen- 
cy, passing through a certain number of layers. In other words, area of cross-phase region lying in the $N$ layers. Of course, you can consider other properties of the volume phase. With the increasing of number of islands in the layer problem is much more complicated. In this paper, we analytically consider the case of one island in the layer and numerically for larger islands and the random number of islands. The distribution function of the phases overlapping area is obtained. Universal behavior of the distribution function is determined.

\section{Statement of the Problem}

Based on the above-described pattern of volume phase formation we observe the stack of circular plates radius $R$, in each of which may be formed round transparent regions. Of course the thickness of plates $h$ we follow will consider negligibly small $h \ll R$. The radius $r$ of these areas random and uniformly distributed in the range of $r_{\min } \div r_{\max }$. Statistical properties of the location of these regions are defined by the distribution function of their centers. For simplicity, we assume that the centers of these areas are uniformly distributed over the plate. In the most general case, the number $n$ of transparent areas on each plate also is random and lies in the range of $n_{\min } \div n_{\max }$. We begin by discussing the simple case when the number of regions $n=1$ on each plate. As a characteristic of the volume phase we choose the intersection of projections of transparent areas in the orthogonal direction to the plates. In other words, this is area of transparent phase, intersecting $n$ layers (see Fig. 1).

\section{One «window» of transparency in each layer}

Suppose that on each of the $N$ plates formed only one area of transparency. Then due to topological reasons, the total "transparency window"in the stack of plates can be only one too. Therefore, we shall be interested in the statistical characteristics of the total area of the transparent phase that passing through the $N$ plates.

We begin with a discussion of exactly solvable case of $N=2$. Let the radii of the transparent areas on the plates are same and equal to $r$. Easy to understand that in this case, the total area of "transparency window" depends only on one parameter - the distance between the centers of the regions.

Consider the case when the transparency region completely located within the plates. For this, the centers of these areas should be placed in the circle of smaller radius $\tilde{R}=R-r$. Thus their centers are uniform distributed in a circle of radius $\tilde{R}$. Denote by $d$ the distance between the centers of projection areas.

Total area of the region of transparency is easy to find for different values of $r$ and $d$ from simple geometrical considerations

$$
s=\left\{\begin{array}{ll}
2 r^{2}\left(\arccos \frac{d}{2 r}-\frac{d}{2 r} \sqrt{1-\left(\frac{d}{2 r}\right)^{2}}\right) & d<2 r \\
0 & d \geq 2 r
\end{array} .\right.
$$

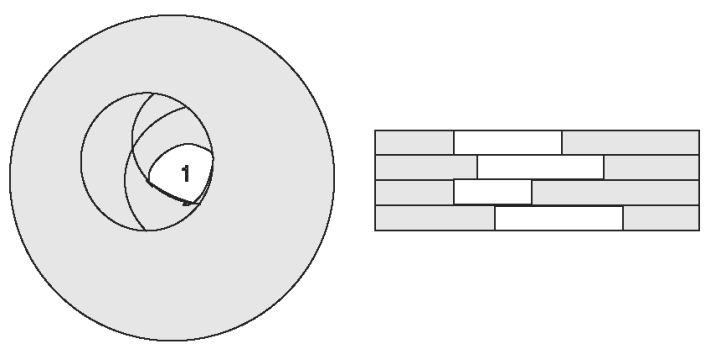

Fig. 1. Shows an example of the intersection of transparent regions in the case of 4 plates and one area of transparency in each layer. At left view from the top, at right side view. The general area of transparency marked by digit 1 . 


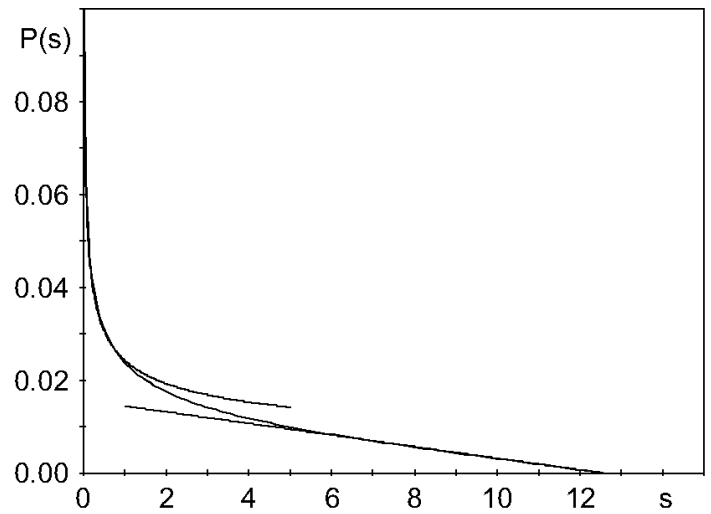

Fig. 2. Shows a typical form of the distribution density of $p_{s}(s)$ with choosing parameters from $r<\tilde{R}$ $(r=2, \tilde{R}=10)$. Also shows - line corresponding large-scale asymptotic behavior $(6)$ and the curve corresponding to the small-scale asymptotic behavior (7).

It is important to emphasize that the function $s(d)$ bijectively and area monotonically decreases with the distance between the centers of the areas on the plates. Therefore, at the certain $p(d)$ - density distribution of the distances between two points uniformly distributed in the disk - density distribution of the total density is easy to get

$$
p_{s}(s)=\left\{\begin{array}{ll}
-p(d(s)) \frac{d}{d s} d(s), & s \in\left(0, \pi r^{2}\right] \\
\operatorname{Pr}(d \geq 2 r) \delta(s), & s=0
\end{array} .\right.
$$

Where $\operatorname{Pr}(d \geq 2 r)$ - the probability that the center of the circles located at a distance greater than $2 r$, i.e. the circles do not intersect. Now, using the well-known results of calculations of the density distribution $p(d)[5]$,

$$
p(d)=\frac{8}{\pi \tilde{R}} \frac{d}{2 \tilde{R}}\left(\arccos \left(\frac{d}{2 \tilde{R}}\right)-\left(\frac{d}{2 \tilde{R}}\right) \sqrt{1-\left(\frac{d}{2 \tilde{R}}\right)^{2}}\right)
$$

it can determine the probability of absence of common region of transparency for two plates

$$
\operatorname{Pr}(d \geq 2 r)=1-\frac{1}{\pi \tilde{R}^{2}}\left(\pi(2 r)^{2}+\left(\tilde{R}^{2}-(2 r)^{2}\right)(\pi-2 \alpha)-\frac{1}{2}\left(2 \tilde{R}^{2}+(2 r)^{2}\right) \sin 2 \alpha\right),
$$

where $\alpha=\arccos \frac{r}{\widetilde{R}}$. This probability becomes zero at $r=R / 2$. Using relation (3) and (4) we obtaine distribution density in form

$$
p_{s}(s)=\left\{\begin{array}{ll}
\frac{8}{\pi \tilde{R}} \frac{d(s)}{2 \tilde{R}}\left(\arccos \left(\frac{d(s)}{2 \tilde{R}}\right)-\left(\frac{d(s)}{2 \tilde{R}}\right) \sqrt{1-\left(\frac{d(s)}{2 \tilde{R}}\right)^{2}}\right)\left(\frac{1}{2 r \sqrt{1-(d(s) / 2 r)^{2}}}\right), & s \in\left(0, \pi r^{2}\right] . \\
2\left(\left(1-\frac{4 r^{2}}{\tilde{R}^{2}}\right)\left(1-\frac{\alpha}{\pi}\right)-\frac{1}{2 \pi}\left(1+\frac{r^{2}}{\tilde{R}^{2}}\right) \sin 2 \alpha\right) \delta(s), & s=0
\end{array} .\right.
$$

Thus the density distribution of the total area of the common region of transparency in the case of the two plates is determined exactly in parametric form by the relation (5). This expression have rather complicated form (see Fig. 2). Therefore, we consider the asymptotic behavior of the distribution density in the region of large values of area $s \approx \pi r^{2}$ and in the low $s \ll \pi r^{2}$. It is easy to get, that in large-scale field

$$
p_{s}(s) \approx \frac{\pi r^{2}-s}{2 \tilde{R}^{2} r^{2}} \quad \text { in region } \quad s \approx \pi r^{2}
$$

Typical form of this asymptotic behavior is shown in Fig. 2 on the background of the corresponding distribution density. Behavior of the distribution density in the small-scale field is much more complex and strongly depends on the parameters $R$ and $r$. So there are three qualitatively different cases: 1) $r<\tilde{R}$ transparency region of small size, 2) $r>\tilde{R}$ large areas of transparency region and the degenerate case 3 ) $r=\tilde{R}$. 


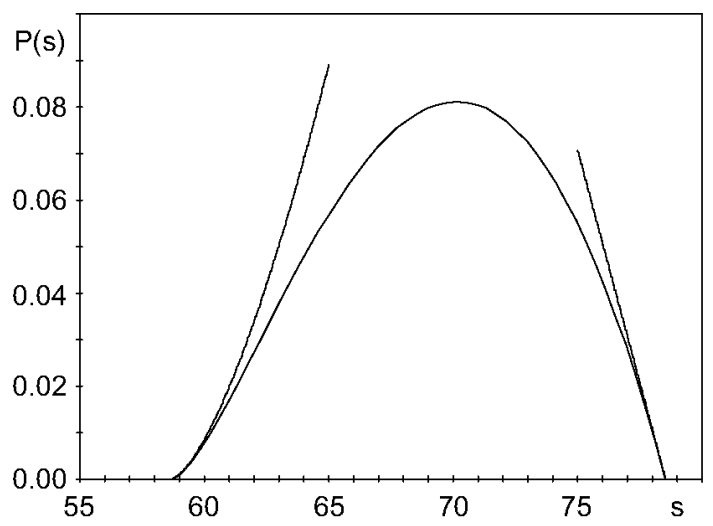

Fig. 3. Density distribution $p_{s}(s)$ in the parameters region $r>\tilde{R}(r=5, \tilde{R}=1)$. Clearly seen asymptotics coincidence (6) and (8), respectively, in large-scale and small-scale regions.

When the condition $r<\tilde{R}$ is satisfied, area where there may be area of transparency large enough. Therefore centers of transparent areas can moves at long distances exceeding $2 r$. In this case, the smallscale region of $\frac{s}{r^{2}} \ll 1$ corresponds to the distance between the centers of the areas of transparency $\frac{d}{2 r} \approx$ 1. Therefore, we introduce a dimensionless small parameter $1-\frac{d}{2 r}=\varepsilon \ll 1$. Using the expansion of relations (1) and (5) on this small parameter it is easy to obtain the asymptotic behavior of small-scale $p_{s}(s)$ in this region of parameters

$$
p_{s}(s) \approx \frac{8}{\pi \tilde{R}} \frac{r}{\tilde{R}}\left(\arccos \left(\frac{r}{\tilde{R}}\right)-\left(\frac{r}{\tilde{R}}\right) \sqrt{1-\left(\frac{r}{\tilde{R}}\right)^{2}}\right) \cdot\left(\frac{1}{2} \sqrt[3]{\frac{13}{12}}\right) \frac{1}{\sqrt[3]{r}} \cdot \frac{1}{\sqrt[3]{s}} \sim s^{-1 / 3} .
$$

It is easy to see that for $r<\tilde{R}$ distribution density $p_{s}(s)$ infinitely increases with decreasing of the $s$. Exact distribution density and asymptotic behavior (7) are shown in Fig. 2.

In the parameter region $r>\tilde{R}$ centers of transparent areas can no longer be at distances greater than $d_{\max }=2 \tilde{R}<2 r$. Naturally, that in these conditions, the area of intersection of the areas of transparency can not be less than

$$
s_{\min }=2 r^{2}\left(\arccos \frac{\tilde{R}}{r}-\frac{\tilde{R}}{r} \sqrt{1-\left(\frac{\tilde{R}}{r}\right)^{2}}\right) .
$$

Therefore, as a dimensionless small parameter should choose $\varepsilon=1-\frac{d}{2 \tilde{R}}$, the smallness of which corresponds to the small-scale field $s \approx s_{\min }$. If $s<s_{\min }$ distribution density is identically zero. Expanding again relations (1) and (5) on this small parameter, we obtain the asymptotic behavior of $p_{s}(s)$ in the parameters region $s \approx s_{\text {min }}$

$$
p_{s}(s) \approx \frac{1}{\pi\left(\tilde{R} r \sqrt{1-(\tilde{R} / r)^{2}}\right)^{5 / 2}}\left(\frac{13}{12 \sqrt{2}}\right) \cdot\left(s-s_{\min }\right)^{3 / 2} .
$$

The typical form of the distribution density in the parameters region $r>\tilde{R}$ corresponding to large-scale (6) and small-scale (8) asymptotics shown in Fig. 3.

We now turn to the degenerate case $r=R$. In this choice of parameters the small-scale region $s \ll 1$ corresponds to the distance between the centers of the areas of transparency $\frac{d}{2 \tilde{R}} \approx 1$. Choosing a small parameter $\varepsilon=1-\frac{d}{2 \tilde{R}}$ as in previous cases easy to get small-scale asymptotics

$$
p_{s}(s) \approx \frac{\sqrt{2}}{\pi \tilde{R} r^{2} \sqrt[3]{r}} \cdot\left(\sqrt[3]{\frac{13 \sqrt{2}}{6}}\right) \cdot s^{2 / 3} .
$$




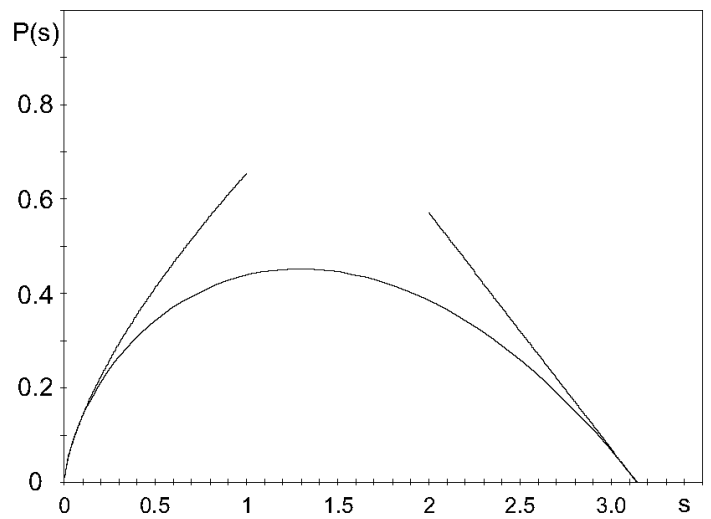

Fig. 4. The distribution density of the total area of transparency in the parameters region $r=\tilde{R}$ $(r=\tilde{R}=1)$. Also shown the asymptotic behavior of $(9)$ and $(6)$.

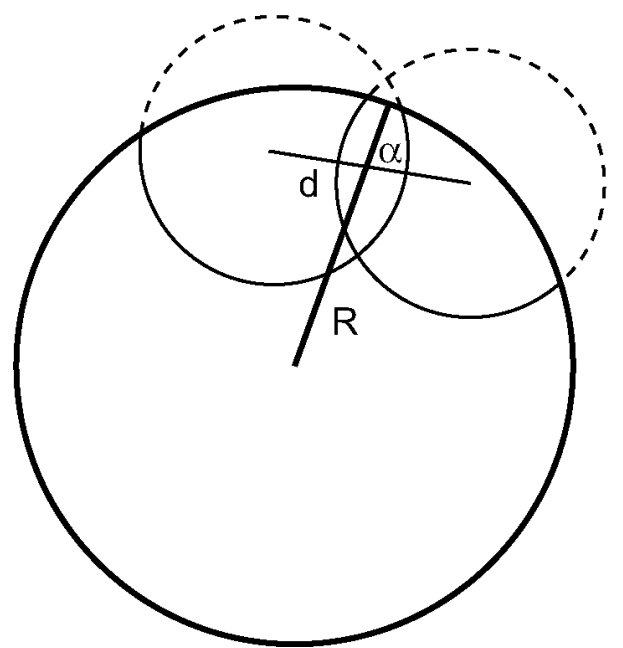

Fig. 5. The intersection of the total «window» of transparency with plate boundary. Angle $\alpha$ between the segment $d$ and radius passing through its middle.

This asymptotic behavior is shown in Fig. 4. Thus in the field of large-scale universal behavior with the asymptotics $(6)$ is observed in all regions of the parameters. Behavior of the distribution density $p_{s}(s)$ in the field of small-scale qualitative changes with parameters $r$ and $R$ changing. This forms three typical power asymptotics.

We now discuss the more complicated case when the transparency region intersect with the boundary of circular plate. In this case, the total transparency window may have a more complex shape due to the intersection with the boundary of the plate. The area of total transparency window becomes a three-parameter (see Fig. 5) in this case. It depends not only on the distance $d$ between the centers of two transparent areas, but also on their location on the plate. Let us take the radius of the plate, that intersectes with the segment $d$ at the midpoint. Then, as the two parameters characterizing the position of two areas, you can choose the distance to the intersection point from plate center and the angle $\alpha$ between radius and $d$ ( see Fig. 5). Analytical study of the density distribution of the total area of transparency becomes too cumbersome. Therefore, the density distribution in this case will be investigated numerically in the next section.

However, some conclusions about the asymptotic form of the distribution density can be made from simple qualitative considerations.

Let's start with a simple case of $r=2 R$. When this condition implemented all the plate consists of a transparent material phase. From physical considerations it can understand that the centers of transparent regions should be on the plate as centers of a new phase. Therefore, the inequality $2 r>R$ is 
not physically implemented. Now consider the case $2 r<R$, when the area of $s$ can be arbitrarily small. In other words, discuss the asymptotic behavior of small-scale. You can expect that it will not change qualitatively. The reason for this connectes from the fact that the total area of transparency with the $s \ll 1$ lies in the interior of a circle of radius $R$ even if centers lie on the boundary of the plates. It is easy to prove that under fairly weak condition $d \geq d_{c}$ intersection of two areas of transparency is always inside of the plate. Critical distance $d_{c}=2 R \sqrt{1-\left(1-\frac{r^{2}}{4 R^{2}}\right)^{2}}$ depends on the size of the plates and the radius of the area of transparency. Then at small sizes of transparency region, part of domains $s$, that undergoing decreasing due to the intersection with the boundary of the plates is small, of the order of $\frac{d_{c}}{2 R} \approx \frac{r}{R}$. It can therefore be expected to remain small-scale asymptotics.

We now turn to the more complex case of a larger number of layers $N>2$. Suppose as before, that on each plate there is one area of transparency with radius $r$. Then for forming in the stack of plates total transparency window with area not less than $s$, the centers of all "windows-should not move away from each other at a distance exceeding some one depending on the value of $s$ (see eg Fig. 1). We denote this distance as $d(s)$.

We introduce the $P(s)$ the probability of the total window area less than $s$, and $P_{d}(d)$ - the probability that the distance between any two centers of windows less than $d$. These probabilities are connected by relation

$$
1-P(s)=P_{d}(d(s))
$$

for $s \in\left[0, \pi R^{2}\right], d \in[0,2 R]$. We define these probabilities using simple considerations. First of all, using dimensional arguments in the most general form, we postulate the dependence of the area $s$ of $d$ and the number of overlapping areas $N$, it can be written as

$$
\frac{s}{d^{2}}=f\left(\frac{r}{R}, \frac{r}{d}, N\right)
$$

where $f\left(\frac{r}{R}, \frac{r}{d}, N\right)$ is explicitly unknown function of the dimensionless parameters. Of course the form of function can be determined for a small number of areas, but it is extremely complicated and cumbersome task. From physical considerations it can determine some of its properties. For example, simple enough to understand that at decreasing of $\frac{r}{R}$ the total area of intersection tends monotonically to zero. It is clear that such natural properties is not enough to calculate the total density distribution $p(s)$. However, as will be shown below, they are enough to get her small-scale asymptotics. To do this, first of all, we note that in the field of small-scale $s / r \ll 1 r / d \rightarrow 1 / 2$. Therefore, the relation (11) in the low $s$ can be simplified by using the approximate dependence $f\left(\frac{r}{R}, \frac{1}{2}, N\right)$. Then, to calculate the distribution density of $p(s)$ let us differentiate relation (9) on $s$

$$
p(s)=\frac{\partial P(s)}{\partial s}=-\frac{\partial P_{d}(d(s))}{\partial d} \frac{\partial d(s)}{\partial s}
$$

, taking into account the dependence (11) with the asymptotic function $f\left(\frac{r}{R}, \frac{1}{2}, N\right)$ for small $s$ we obtain the asymptotic small-scale behavior of form

$$
p(s) \sim \frac{1}{\sqrt{s}} .
$$

The main hypothesis is the absence of the vanishing of $\frac{\partial P_{d}(d(s))}{\partial d}$ for small $s$ or $d$ tends to the critical value. Of course, these qualitative considerations are verified by numerical simulations follow.

\section{Random number of "transparency windows" in each layer}

We now turn to modeling the more general case. Suppose that on each plate may appear many areas of transparency. Physically, this is a more realistic case. To do this, some number of transparent areas of the same radius $r$ was formed randomly on each plate. The centers of these regions were assumed uniformly distributed on the plate. Distribution of number of areas on a separate plate $n$ was uniformly 
distributed in the range of $n \in[5, \ldots, 20]$. Further we calculated the total area of the intersection areas of transparency throughout the stack of circular plates of radius $R$. Selection the number of plates in the stack is limited by two cases $N=3$ and $N=4$. With an increasing of number of plates in the stack overall area of intersection of transparency zones $s$ becomes small. The latter condition makes it necessary to substantially increase the number of experiments in order to reduce fluctuations of $s$ and therefore these cases not considered. For each $N 6$ series with 500 numerical experiments was made. Each series was determined by choise of the value of $r$. Value $r$ aref $r=0.1 R ; 0.14 R ; 0.18 R ; 0.22 R ; 0.26 R ; 0.3 R$. Example of the common areas of transparency, formed at the conditions described above for the case of $N=3$ is shown in Fig. 6. You may notice that typical in this case is the collision pairs and triples of transparent

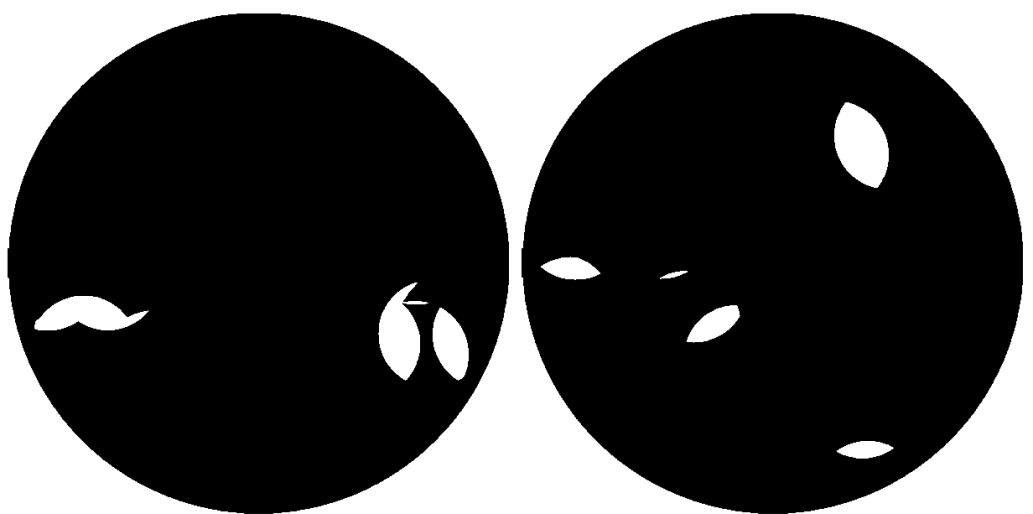

Fig. 6. Shows a typical example of transparency «windows», forming in a numerical experiment for the number of plates $N=3$ and $r=0.22 R$.

areas. In other words, the probability dominates for formation of common areas of transparency due to intersection of number of areas equal to the number of plates in the stack. Physically it is performed under the condition if the total area of all areas of transparency is small compared with the area of the plate.

With decreasing number of domains $n \leq 3$ probability of total transparency window formation becomes small and in almost all experiments stack plates have no common transparency windows. It obtaines opaque samples. In the opposite case, the increase of the number $n>20$ transparent areas on each plate, leads to the formation of the common areas of transparency with complex cluster shape (see Figure 7), up to the formation of a single cluster, filling the entire area of the stack of plates. The reason for this is increase of the total area of transparent zones with increase $n$, which becomes comparable to or greater than the plate area. Under such conditions, the formation of the general transparency window happenes due to the intersection of a large number of areas - number significantly exceeds the number of plates in the stack.

In numerical simulation we determined distribution density of common areas of transparency zones. Such distribution densities were constructed for each of the values of $r$. Fig. 8 shows a histogram of distribution densities in logarithmic scale for the cases $N=3$ and $N=4$. Each character set corresponds to a value of radius $r$ of transparent areas. It is easy to notice the presence of a linear part of the dependence of the density distribution of $s$. For convenience, this dependence is shown for a one value $r$ in Fig. 8 by line. This means that there is a universal asymptotics of small-scale field of form

$$
p(s) \sim s^{-0.55} \approx 1 / \sqrt{s} .
$$

Thus numerical simulation confirms the universal small-scale asymptotics (12), obtained from the qualitative considerations in the previous section.

However, in the more realistic case the transparency regions in the layer can have different sizes $r$. Therefore it is interesting to find out what distribution $p(s)$ is in a random distribution of radii of transparency zones in each layer. For this numerical experiments were carried with a random distribution of $r$ with arbitrary values in the range $[0.1 R, \ldots, 0.3 R]$. For simplicity, the values of $r$ assumed to be 


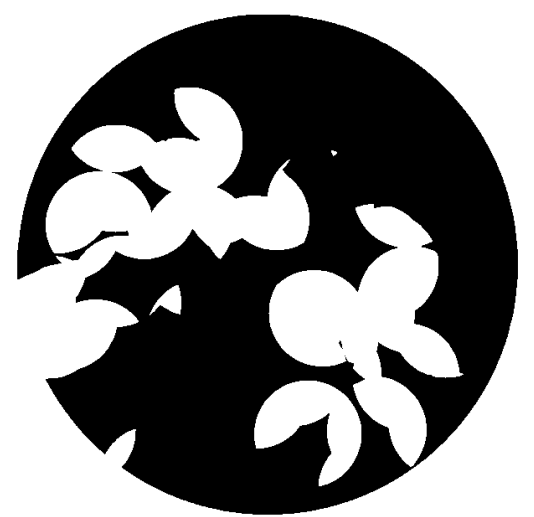

Fig. 7. Shows the implementation of forming of two major disconnected clusters for $n=30, N=3$ and $r=0.22 R$. With increasing $n$ typically it can see merging of these clusters into a single cluster.
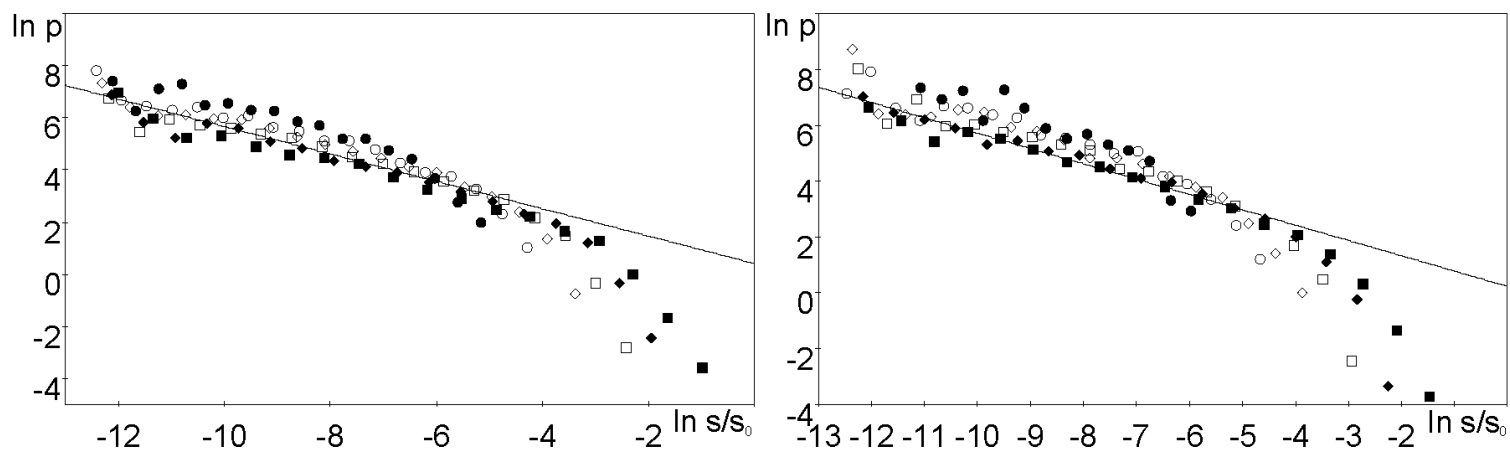

Fig. 8. Distribution density of area $s$ of transparency «windows». Shows the results of numerical experiments with fixed radii $r$ of transparency regions on the plates for $N=3$ left, for $N=4$ on the right. Values of distribution densities for different radii $r$ are marked as follows: $\bullet-r=0.1 R, \circ-r=0.14 R$, $\diamond-r=0.18 R, \square-r=0.22 R,-r=0.26 R, \boldsymbol{\square}-r=0.3 R$. Line corresponding to the asymptotic behavior of (12), to avoid cluttering the figure, held only for the data marked with the symbol $\square$.

uniformly distributed in the interval. Number of areas on each plate was, as above, random umber in the range $n \in[5, \ldots, 20]$ with the same distribution. Numerical experiments were carried out for the same number of plates in the stack as in the previous experiments, $N=3$ and $N=4$. For each value of $N$ was made 500 experiments. The averaged results are shown in Fig. 9. For clearness, in Fig. 9 we show a line, corresponds to small-scale asymptotic behavior (12).

Thus in all more realistic cases in small-scale region the universal asymptotics (12) observed.
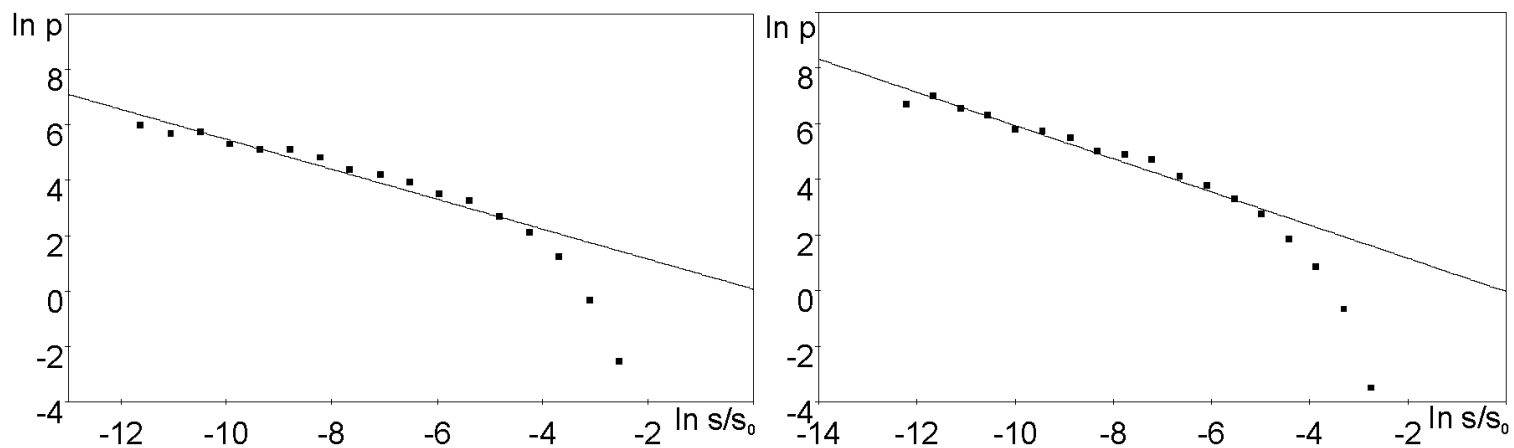

Fig. 9. Distribution density of area $s$ of transparency «windows», the results of numerical experiments with variable radius $r$ of regions on the plates for $N=3$ on the left, for $N=4$ on the right. Straight line corresponds to the small-scale asymptotic behavior (12). 


\section{References}

1. V.I.Trofimov, V.A.Osadchiy, Growth and Morphology of Thin Films, Energoatomizdat, Moscow (1993) [in Russian].

2. B.Lewis, J.C.Anderson, Nucleation and Growth of Thin Films, Academic Press, New York (1978)

3. K.Oura, V.G.Livshits, A.A.Saranin, et al., Introduction to Surface Physics, Nauka, Moskow (2006) [in Russian].

4. V.Barton, B.Kabrera, F.Frank, Elementary Processes of Crystal Growth, IIL, Moscow (1959) [in Russian].

5. M.Kendall, P.Moran, Geometric Probabilities, Nauka, Moscow (1972) [in Russian]. 